\title{
SECaps: A Sequence Enhanced Capsule Model for Charge Prediction
}

\author{
Congqing $\mathrm{He}^{1}$, Li Peng ${ }^{1(凶)}$, Yuquan $\mathrm{Le}^{1}$, Jiawei He ${ }^{1}$, and Xiangyu Zhu ${ }^{2}$ \\ 1 College of Computer Science and Electroic Engineering, Hunan University, China \\ 2 JD Digits, China \\ \{hecongqing, $r j \_l$ peng, leyuquan, hejiawei\}@hnu.edu.cn \\ zhuxiangyu3@jd.com
}

\begin{abstract}
Automatic charge prediction aims to predict appropriate final charges according to the fact descriptions for a given criminal case. Automatic charge prediction plays a critical role in assisting judges and lawyers to improve the efficiency of legal decisions, and thus has received much attention. Nevertheless, most existing works on automatic charge prediction perform adequately on highfrequency charges but are not yet capable of predicting few-shot charges with limited cases. In this paper, we propose a Sequence Enhanced Capsule model, dubbed as SECaps model, to relieve this problem. Specifically, following the work of capsule networks, we propose the seq-caps layer, which considers sequence information and spatial information of legal texts simultaneously. Then we design a attention residual unit, which provides auxiliary information for charge prediction. In addition, our SECaps model introduces focal loss, which relieves the problem of imbalanced charges. Comparing the state-of-the-art methods, our SECaps model obtains $4.5 \%$ and $6.4 \%$ absolutely considerable improvements under Macro F1 in Criminal-S and Criminal-L respectively. The experimental results consistently demonstrate the superiorities and competitiveness of our proposed model.
\end{abstract}

Keywords: Charge prediction $\cdot$ Capsule networks $\cdot$ Few-shot $\cdot$ Focal loss.

\section{Introduction}

The task of automatic charge prediction is to help lawyers or judges to determine appropriate charges (e.g., fraud, robbery or larceny) according to a given case. The automatic charge prediction plays a critical role in many legal intelligent scenarios (e.g., legal assistant systems or legal consulting). The legal assistant system can improve the efficiency of professionals. The legal consulting is benefit for people who are unfamiliar with legal terminology of their interested cases. Therefore, automatic charge prediction is an extremely beneficial topic for many legal intelligent scenarios.

Most existing works of automatic charge prediction can be divided into three categories. The first categories are usually mathematical or quantitative [8]17], which are restricted to a small dataset with few labels. The second categories use a lot of manpower to design legal text features, and then the machine learning algorithms are applied. For example, Liu et al. [12] utilize word-level and phrase-level features and K-Nearest 
Neighbor (KNN) method to predict charges. Liu et al. [13] use Support Vector Machine (SVM) for preliminary article classification, and then re-rank the results by using word level features and co-occurence tendency among articles. Katz et al. [4] extract efficient features from case profiles (e.g., dates, locations, terms, and types). However, the shallow textual features of human designs require a lot of manpower and have limited ability to capture the semantic information of legal texts. Recently, owing to the success of deep neural networks on nature language processing tasks [9], some popular neural network methods apply on automatic charge prediction task [14]3], obtaining attractive performance. For example, Luo et al. [14] propose an attention-based neural network for charge prediction by incorporating the relevant law articles. This work is not yet capable of predicting few-shot charges with limited cases. Hu et al. [3] propose attribute-attentive charge prediction model to alleviate few-shot charges problem. In the other hand, Zhao et al. [24] apply the capsule network [19] to the text classification scene and achieve attractive performance.

Inspired by the above observations, in this paper we propose a Sequence Enhanced Capsule model, dubbed as SECaps model. The SECaps model proposes the seq-caps layer, which can consider sequence information and spatial information of legal texts simultaneously. Then the model designs a attention residual unit, which can provide auxiliary information for charge prediction. In addition, the model introduces focal loss, which can relieve the problem of imbalanced charges.

To summarize, the main contributions of this paper are:

- We propose a Sequence Enhanced Capsule model that not only considers sequence information and spatial information of legal texts simultaneously, but also has a competitive performance on the problem of few-shot charges.

- Our SECaps model introduces focal loss, which first appear on object detection problems and is able to alleviate the problem of imbalanced charges to some extent.

- Comparing the state-of-the-art methods, our SECaps model achieves $4.5 \%$ and $6.4 \%$ absolutely considerable improvements under Macro F1 in Criminal-S and Criminal$\mathrm{L}$ respectively. The experimental results consistently demonstrate the superiorities and competitiveness of our proposed model.

\section{Model}

\subsection{Capsule Network}

Capsule network proposed by Sabour et al. [19] has shown strong competitiveness in the field of images. Capsule network adopts dynamic routing mechanism, and routes the lower-level capsules to higher-level capsules.

Define lower-level capsules as $u=\left(u_{1}, u_{2}, \cdots, u_{n}\right)$ and higher-level capsules as $v=$ $\left(v_{1}, v_{2}, \cdots, v_{m}\right)$, where $u_{i} \in R^{d}$ is the $i$-th capsule in lower-level and $v_{j} \in R^{p}$ is the $j$-th capsule in higher-level, $d$ and $p$ represent the dimension of lower-level capsules and the dimension of higher-level capsules respectively. The dynamic routing mechanism have the follows two steps:

- Linear transformation. In this step, an intermediate feature vector $u_{j \mid i}$ of $u_{i}$ is produced by multiplying the output $u_{i}$ by a weight matrix $W_{i j}$.

$$
u_{j \mid i}=W_{i j} u_{i} .
$$


Where $W_{i j}$ is a weight matrix which connects between lower-level $u_{i}$ and higher-level $v_{j}$. There are $n \times m$ weight matrices $W_{i j}$ between two capsule layers. However, the weight matrices $W_{i j}$ produces the large amount of the parameters. In order to reduce parameters, we introduce share weight mechanism, which is similar with Zhao et al. [24]. In share weight mechanism, the connection between all the lower-level capsules and the $j$-th capsule in higher-level share a common weight matrix $W_{j}$, so the intermediate feature vector $u_{j \mid i}$ is computed as follows

$$
u_{j \mid i}=W_{j} u_{i} .
$$

- Clustering for lower-level capsules. In this step, the dynamic routing mechanism minimizes an agglomerative fuzzy k-means clustering-like loss function as follows:

$$
\begin{aligned}
& \min _{C, S}\left\{\operatorname{loss}(C, S)=-\sum_{i, j} c_{i j}\left\langle u_{j \mid i}, v_{j}\right\rangle+\alpha \sum_{i, j} c_{i j} \log c_{i j}\right\} \\
& \text { s.t. } c_{i j}>0, \sum_{j=1}^{n} c_{i j}=1,\left\|v_{j}\right\| \leq 1
\end{aligned}
$$

where $C=\left[c_{i j}\right]$ is an $n$-by- $m$ partition matrix, $c_{i j}$ represents the association degree of $i$-th lower-level capsule $u_{j \mid i}$ to the $j$-th cluster $s_{j}, S=\left[s_{1}, s_{2}, \cdots, s_{m}\right]^{T}$ is $m$ cluster centers. Then, similar to Hinton et al. [19], we use a non-linear "squashing" function to ensure that short vectors get shrunk to almost zero length and long vectors get shrunk to a length slightly below 1 , and thus get the higher-level capsule. The formula of "squashing" is described as follows:

$$
\operatorname{squash}\left(s_{j}\right)=\frac{\left\|s_{j}\right\|^{2}}{1+\left\|s_{j}\right\|^{2}} \frac{s_{j}}{\left\|s_{j}\right\|} .
$$

Deriving the coordinate descent updates of $C$ and $S$, we obtain the updates in Algorithm 1 [23].

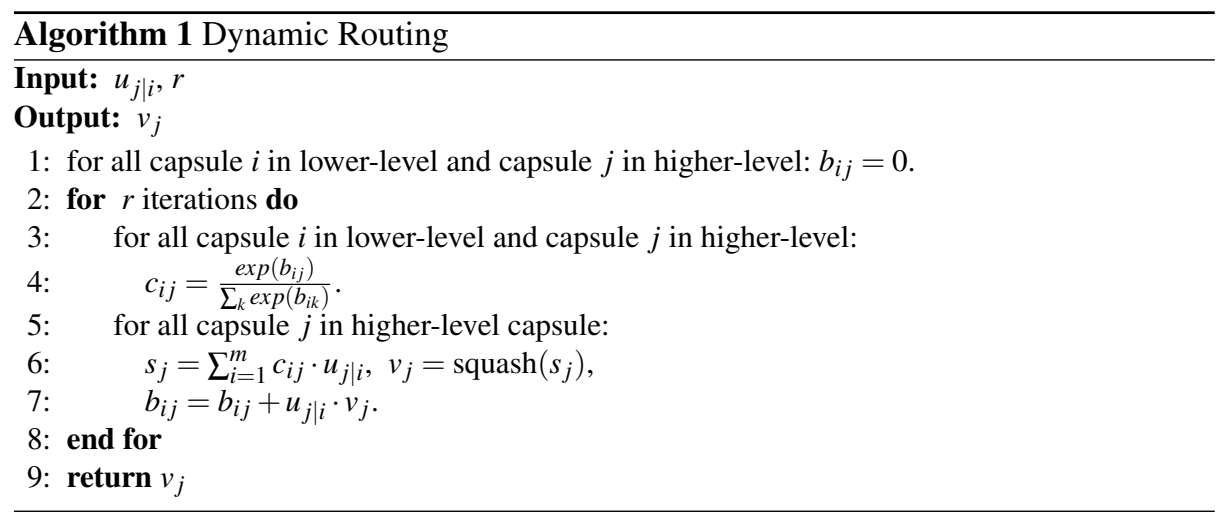

\subsection{Seq-caps Layer}

Capsule networks treat a feature as a activity vector, it can be used in many nature language processing (NLP) tasks [24] . Generally, the input of many NLP tasks is a 


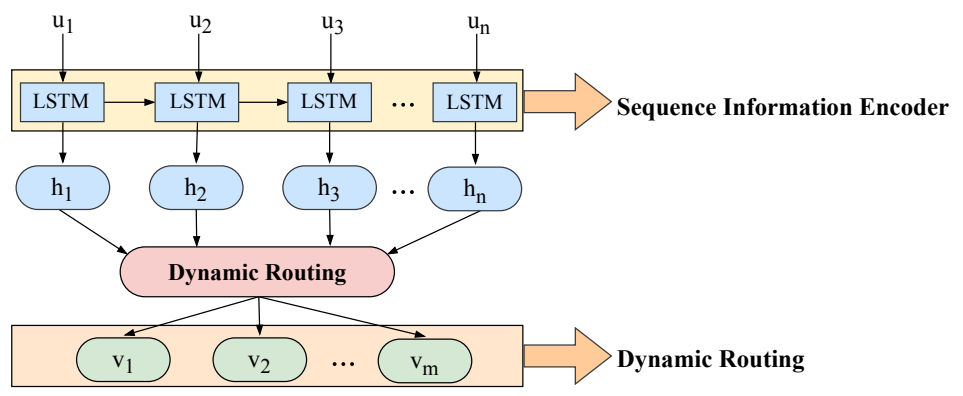

Fig. 1. The framework of seq-caps layer, the input capsules $u=\left\{u_{1}, u_{2}, \cdots, u_{n}\right\}$ is lower-level capsules and the output capsules of the seq-caps layer is $v=\left\{v_{1}, v_{2}, \cdots, v_{m}\right\}$.

sequence of words which represents a sentence or a text. Each word of the sequence is often transformed into the distributed representation of word, due to the success of word embeddings [16]. The word distributed representation can be seen as a activity vector, and thus a sequence of words can be seen as a group of capsules. We can use capsule networks in these NLP tasks as long as we set the first layer of capsule networks to words distributed representation of words sequence.

However, the higher-level capsules capture the key information of lower-level capsules by making use of fuzzy clustering. This lead to the higher-level capsules loss the sequence information of the input word sequence. In fact, a word is often highly correlated with its context. Losing sequence information weaken the performance of capsule network in NLP tasks. Therefore, we propose a new basic structure, named seq-caps layer, to enhance the capsule layer by taking the sequence information into account.

Figure 1 shows the framework of our seq-caps layer. Suppose the input capsules of the seq-caps layer is $u=\left\{u_{1}, u_{2}, \cdots, u_{n}\right\}$, our seq-caps layer has the follows two component:

- Sequence Information Encoder. It uses a Long Short-Term Memory [2] (LSTM) encoder as a sublayer to restore sequence information of the input capsules. In this step, we get hidden layer $h_{1}, h_{2}, \cdots, h_{n}=\operatorname{LSTM}\left(u_{1}, u_{2}, \cdots, u_{n}\right)$.

- Dynamic Routing. It transforms the hidden layer to higher-level capsules by using dynamic routing mechanism (Algorithm 11. In this step, we get higher-level capsules $\left\{v_{1}, v_{2}, \cdots, v_{m}\right\}$.

\subsection{SECaps Model}

In charge prediction task, the fact description of a case can also be seen as a sequence of words $x=\left\{x_{1}, x_{2}, \cdots, x_{n}\right\}$, where $n$ is length of the fact description, $x_{i}$ is a word. Given the fact description $x$, the charge prediction task aims to predict a charge $y \in Y$ from a charge set $Y$.

In real world, some charges (e.g., theft, intentional injury) have large amount of cases, while others like scalping relics, disrupting the order of the court just have few cases. This is so called few-shot problem. Traditional models pay much attention to 


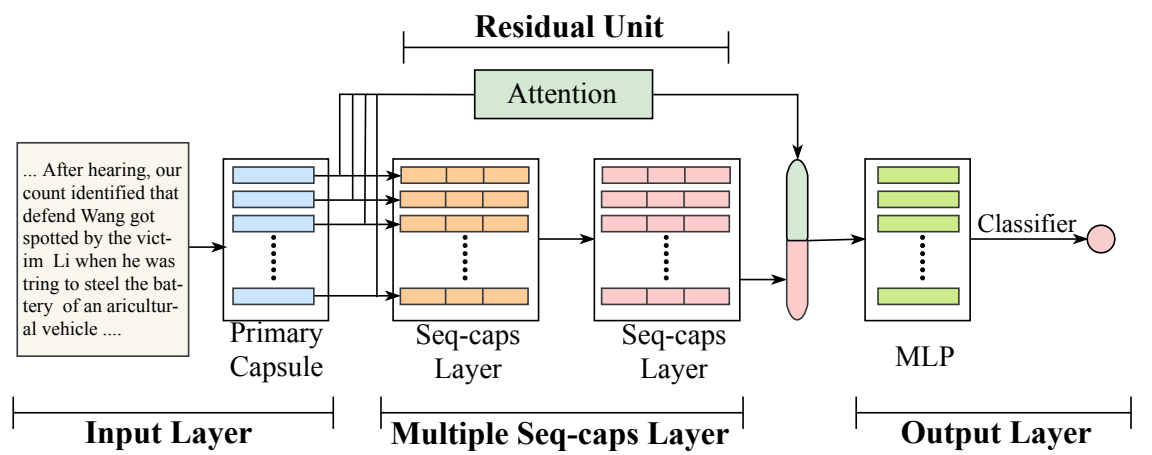

Fig. 2. The architecture of SECaps model, including Input layer, Multiple seq-caps layer, Attention, and Output layer.

charges which have large amount of cases and thus ignore these few-shot charges. In order to mitigate the effect of few-shot problem, our SECaps model combines seq-caps layer with the focal loss [10], in which, seq-caps layer captures the prominent features and the semantic information of legal texts in a better way and focal loss is able to alleviate category imbalances to some extent.

Our SECaps model includes four parts: Input layer, Multiple seq-caps layer, Attention, Output layer. Figure 2 show the architecture of our SECaps model.

Input layer: In this part, we treat the fact description of a case as a sequence of words $x=\left\{x_{1}, x_{2}, \cdots, x_{n}\right\}$, then, each word of the sequence is transformed to primary capsule.

Multiple seq-caps layer: This part has two seq-caps layers. We treat the word embeddings as primary capsules, and then transfer primary capsules to higher-level capsules. The seq-caps layer outputs advanced semantic representation which are captured from fact description of a case. Meanwhile, seq-caps layer restores the sequence information of fact description, which is key factor for charge prediction.

Attention: When the multiple seq-caps layer aggregates primary capsules into higherlevel capsules, the model only focuses on the most important legal case's information. The similar as $\mathrm{He}$ et al. [1] , we propose a new residual unit to improve the generalization and provide auxiliary information for charge prediction. Our model introduces attention mechanism as the residual unit, to encode the primary capsule which can capture the global context information. Suppose the primary capsules from the input part is $\left\{t_{1}, t_{2}, \cdots, t_{n}\right\}$, the residual unit's vector $c$ is computed as follows:

$$
\begin{aligned}
& e_{i}=\tanh \left(W t_{i}+b\right) \\
& \alpha_{i}=\frac{\exp \left(e_{i}\right)}{\sum_{j} \exp \left(e_{j}\right)} \\
& c=\sum_{i} \alpha_{i} t_{i}
\end{aligned}
$$


where $W$ is a weight matrix and $b$ is bias.

Output layer: In order to consider prominent features and the global context information together, we first flatten all the feature vectors from the Multiple seq-caps layer, and concatenate with the global context vector $c$. Then, we use a fully connected network and softmax function to generate the probability $y=\left(y_{1}, y_{2}, \cdots, y_{k}\right)$, where $k$ is the number of charge. As for loss function, we apply focal loss to SECaps model. Focal loss is proposed for dense object detection initially, which address the few-shot problem by reshaping the standard cross entropy loss such that it down-weights the loss assigned to well-classified examples [10]. It can be calculate as follows:

$$
F L\left(y_{t}\right)=\alpha_{t}\left(1-y_{t}\right)^{\gamma} \log \left(y_{t}\right)
$$

where $y_{t}$ is the $t$-th output of $y, \alpha_{t} \in[0,1]$ is weighting factor and $\gamma$ is the focusing parameter.

\section{Experiments}

\subsection{Dataset and Evaluation Metrics}

Datasets We employ three public dataset ${ }^{3}[3]$ for charge prediction, which was published by the Chinese government from China Judgments Online ${ }^{4}$ For each case in these datasets are constituted by several parts, such as fact, charges, and attributions. Three datasets which contain the same number of charges but the different number of scales, named as Criminal-S (small), Criminal-M (medium) and Criminal-L (large). The statistics of our datasets are reported in Table 1 .

Table 1. The statistics of different datasets

\begin{tabular}{c|ccc}
\hline Datasets & Criminal-S & Criminal-M & Criminal-L \\
\hline train & 61,589 & 153,521 & 306,900 \\
valid & 7,755 & 19,250 & 38,429 \\
test & 7,702 & 19,189 & 38,368 \\
\hline
\end{tabular}

Evaluation Metrics Following previous works on charge prediction [14]3], we employ Accuracy (Acc.), Macro Precision (MP), Macro Recall (MR) and Macro F1 (MF) as our main evaluation metrics.

\subsection{Baselines}

The same as $\mathrm{Hu}$ et al. [3] , we select several representative text classification models and two state-of-the-art methods for charge prediction as baselines.

3 https://thunlp.oss-cn-qingdao.aliyuncs.com/attribute_charge.zip

4 http://wenshu.court.gov.cn 
TFIDF+ SVM is a simple machine learning model based on Support Vector Machine (SVM) [21] with linear kernel, extracting text features from term-frequency inverse document frequency (TFIDF) [20] as input. Then two based deep learning model are also to compare with our SECaps model, the first is Convolutional Neural Network (CNN) [6] which is to encode fact descriptions with multiple filter widths, and the second employs a two-layer LSTM [2] with a max-pooling layer as the fact encoder.

Moreover, to future illustrate the effectiveness of our model, we compared our model with two latest similar tasks, Fact-Law Attention Model [14] and Attributeattentive Charge Prediction Model [3]. Fact-Law Attention Model is an attention-based neural network method for charge prediction task, and $\mathrm{Hu}$ et al. [3] propose Attributeattentive Charge Prediction Model which can infer the attributes and charges simultaneously.

\subsection{Experiment Settings}

Since all the case documents have been employed THULAC 5 for word segmentation and set each document maximum length to 500. For the TFIDF+SVM, the experiment is established by extracting the feature size to 2,000 and using SVM with linear kernel for training. Moreover, to make a fair comparison, we establish a set of neural models. We employ word2vec [16] for word embedding with size to 100 before the experiment. For the CNN and the LSTM, by setting the filter widths to $\{2,3,4,5\}$ with each filter size to 25 for consistency and the hidden state size to 100 of LSTM respectively. What's more, two recent models which proposed by Luo et al. [14] and Hu et al. [3] respectively, the parameters remain the same as the original paper.

Our SECaps model uses the Adam [7] optimization method to minimize the focal loss [10] over the training data. For hyperparameters of Adam and focal loss, we keep it consistent with the original papers since better performance in their papers. Our SECaps model has two seq-caps layers, and we set different hyperparameters which is shown in Table 2. Then our model utilizes two fully connected layers by setting to $1024 \times 512$.

Table 2. Hyperparameters for two seq-caps layers. CapsNums, CapsDims, Routing and Hidden units represent the number of capsules, the dimension of capsules, the number of dynamic routing and the hidden units of LSTM respectively .

\begin{tabular}{c|cccc}
\hline Layer & CapsNums & CapsDims & Routing & Hidden units \\
\hline seq-caps layer 1 & 10 & 16 & 5 & 200 \\
\hline seq-caps layer 2 & 5 & 10 & 5 & 128 \\
\hline
\end{tabular}


Table 3. Charge prediction results of three datasets

\begin{tabular}{c|cccc|cccc|cccc}
\hline Datasets & \multicolumn{4}{|c|}{ Criminal-S } & \multicolumn{4}{c|}{ Criminal-M } & \multicolumn{4}{c}{ Criminal-L } \\
\hline Metrics & Acc. & MP & MR & MF & Acc. & MP & MR & MF & Acc. & MP & MR & MF \\
\hline TFIDF+SVM & 85.8 & 49.7 & 41.9 & 43.5 & 89.6 & 58.8 & 50.1 & 52.1 & 91.8 & 67.5 & 54.1 & 57.5 \\
CNN & 91.9 & 50.5 & 44.9 & 46.1 & 93.5 & 57.6 & 48.1 & 50.5 & 93.9 & 66.0 & 50.3 & 54.7 \\
CNN-200 & 92.6 & 51.1 & 46.3 & 47.3 & 92.8 & 56.2 & 50.0 & 50.8 & 94.1 & 61.9 & 50.0 & 53.1 \\
LSTM & 93.5 & 59.4 & 58.6 & 57.3 & 94.7 & 65.8 & 63.0 & 62.6 & 95.5 & 69.8 & 67.0 & 66.8 \\
LSTM-200 & 92.7 & 60.0 & 58.4 & 57.0 & 94.4 & 66.5 & 62.4 & 62.7 & 95.1 & 72.8 & 66.7 & 67.9 \\
\hline Fact-Law Att. [14] & 92.8 & 57.0 & 53.9 & 53.4 & 94.7 & 66.7 & 60.4 & 61.8 & 95.7 & 73.3 & 67.1 & 68.6 \\
Attribute-att. [3] & 93.4 & 66.7 & 69.2 & 64.9 & 94.4 & 68.3 & 69.2 & 67.1 & 95.8 & 75.8 & 73.7 & 73.1 \\
\hline SECaps Model & $\mathbf{9 4 . 8}$ & $\mathbf{7 1 . 3}$ & $\mathbf{7 0 . 3}$ & $\mathbf{6 9 . 4}$ & $\mathbf{9 5 . 4}$ & $\mathbf{7 1 . 3}$ & $\mathbf{7 0 . 2}$ & $\mathbf{6 9 . 6}$ & $\mathbf{9 6 . 0}$ & $\mathbf{8 1 . 9}$ & $\mathbf{7 9 . 7}$ & $\mathbf{7 9 . 5}$ \\
\hline
\end{tabular}

\section{Results and Analysis}

\subsection{Performance Comparison}

Table 3 shows the results of our model with baselines on three datasets. Overall, we find that the SECaps model outperforms all previous baselines with a significant margin on three datasets. More specifically, compared to the previous state-of-the-art in charge prediction [3], our model obtains $4.5 \%, 2.5 \%$, and $6.4 \%$ absolutely considerable improvements across three datasets respectively under MF, which demonstrates that the effectiveness of our SECaps model for charge prediction. This trend suggests that SECaps is capable of capturing advanced semantic representation of legal texts which are crucial for charge prediction.

We propose a novel layer, termed seq-caps, which considers sequence information and spatial information of legal texts simultaneously. Then our SECaps model employs multiple seq-caps layer to capture sequence information and advanced semantic representation which has a significant impact for charge prediction. In addition, our SECaps model introduces residual unit and designs an attention mechanism to capture significant auxiliary information of primary capsule for charge prediction. Consequently, our SECaps model obtains state-of-the-art performance on three datasets without any additional ancillary information.

\subsection{Few-shot Charges Comparison}

Table 4. Macro F1 values of various charges on Criminal-S

\begin{tabular}{c|ccc}
\hline Charge Type & Low-frequency & Medium-frequency & High-frequency \\
\hline Charge Number & 49 & 51 & 49 \\
\hline LSTM-200 & 32.6 & 55.0 & 83.3 \\
Attribute-att. [3] & 49.7 & 60.0 & 85.2 \\
\hline SECaps Model & $\mathbf{5 3 . 8}$ & $\mathbf{6 5 . 5}$ & $\mathbf{8 9 . 0}$ \\
\hline
\end{tabular}

\footnotetext{
5 https://github.com/thunlp/THULAC-Python
} 
Following Hu et al. [3], to further illustrate the effectiveness of the SECaps model on handling few-shot charges, we run a set of experiments to split charges with different frequency. We divide the charges into three parts according to the frequencies (lowfrequency, medium-frequency and high-frequency). Low-frequency is defined as the charges appears less than 10 times (includes 10 times) on Criminal-S, high-frequency is defined as the charges appears more than 100 times (excepts 100 times) on Criminal-S and otherwise belongs to medium-frequency.

Table 4 shows the performance of our SECaps model with different frequency on Criminal-S, we report the low-frequency, the medium-frequency and the high-frequency results of MF. From the table we see that the MF of low-frequency is 53.8\% which achieves more than 65\% improvements than LSTM-200 and obtains a considerable improvement by $4.1 \%$ over the state-of-the-art baseline [3]. Our SECaps model proposes a seq-caps layer, which can capture advanced semantic representation, and thus relieve the problem of insufficient features in the few-shot charge prediction. Specifically, the SECaps model has good power on vector representation and time series representation ability, focal loss has a good performance in handling the problem of unbalanced classification, which can make up for lack of the unbalanced classification problem.

\subsection{Ablation Studies}

Table 5. Ablation studies comparing our SECaps model with different residual units on CriminalS.

\begin{tabular}{c|cccc}
\hline Models & Acc. & MP & MR & MF \\
\hline SECaps Model & $\mathbf{9 4 . 8}$ & $\mathbf{7 1 . 3}$ & $\mathbf{7 0 . 3}$ & $\mathbf{6 9 . 4}$ \\
\hline SECaps w/o Attention & 94.7 & 67.7 & 68.1 & 66.4 \\
SEcaps with Added Unit & 94.6 & 66.1 & 65.3 & 64.0 \\
\hline
\end{tabular}

In order to evaluate the residual unit for the influence of our SECaps model, we conduct a series of ablation studies among various approaches. Table 5 shows the results of various variant approaches.

- SECaps w/o Attention which employs only two seq-caps layers to encode the primary capsules provides less performance when compared to our SECaps model. This signifies primary capsules aggregate higher-level capsules which only focuses on the most important legal cases information. It demonstrates that the residual unit is able to improve the generalization. Beside, it prove that the residual unit can provide auxiliary information for charge prediction.

- SEcaps with Added Unit which employ simply added primary capsule unit instead of attention unit. As can be seen in Table 5, our SEcaps with added unit model even worse than SECaps w/o Attention model. This phenomenon shows that the model brings some noise information if employ simply added primary capsules as the residual unit. Overall, attention unit can pay attention to the important information of primary capsules for charge prediction, which reinforce the SECaps model for capturing critical evidence. 


\subsection{Impact of Hyperparameter}

In this section, we first study how the number of capsules affect the performance on Criminal-S. Our SECaps model are set the number of capsules from 7 to 12 in the seqcaps layer 1 and retained the rest of parameters unchanged. As shown in Figure 3(a), we find that our SECaps model adds more capsules which can capture more vector representation. However, more capsules introduce noise which consequently decrease accuracy. We set the parameter CapsNums $=10$ in seq-caps layer 1 to balance the ability of representation of higher-level capsules.

We also study how the dimension of capsules affect the performance on CriminalS. As shown in Figure 3(b) We find that our SECaps model obtains the state-of-theart performance when dimension is set to 16 . The results indicate larger dimension's capsule is contributing to improve the performance. However, when the dimension of capsules is too large, the model aggregates more information from primary capsules and brings noise information which is helpless for charge prediction. Therefore, the dimension of capsules should not be too large.

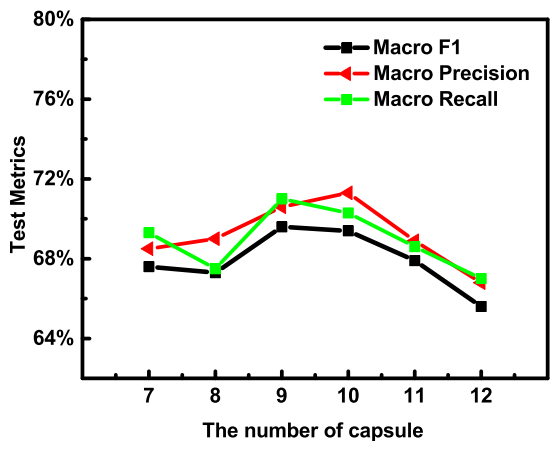

(a) The number of capsule.

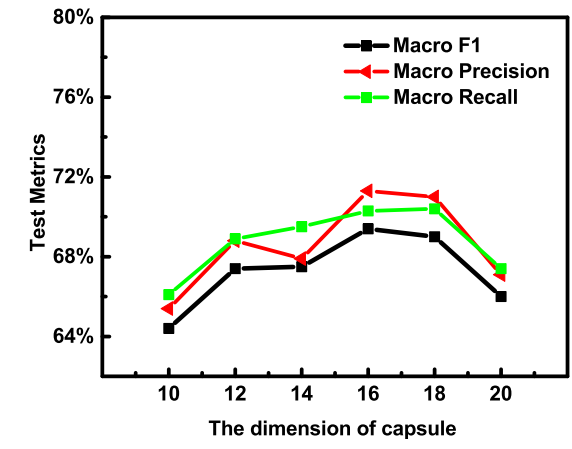

(b) The dimension of capsule.

Fig. 3. Fig 3(a) describes the relationship between $M P, M R, M F$ and the number of capsules in seq-caps layer 1. Fig 3(b) describes the relationship between MP,MR,MF and the dimension of capsules in seq-caps layer 1 .

\section{Related Works}

Automatic charge prediction plays an important role in the legal area and thus has received much attention. Researchers have proposed many methods for implementing automatic charge prediction. In this paper, these methods are classified into three categories: (1) traditional methods, (2) machine learning methods, and (3) deep neural network methods.

Traditional methods are usually mathematical or quantitative. Kort [8] represents an attempt to apply quantitative methods to the prediction of human events. Nagel [17] 
applys correlation analysis to case prediction. Keown [5] introduces mathematical (e.g., linear models and the scheme of nearest neighbors) models, which is used for legal prediction. These traditional methods have achieved some effects in certain scenarios, but they are restricted to a small datasets with few labels.

Researchers begin to use machine learning methods to handle charge prediction because of its success in many areas. This type of work usually focuses on extracting features from case facts and then using machine learning algorithms to make predictions. Liu et al. [11|12] use K-Nearest Neighbor (KNN) method to classify criminal charges. Lin et al. [22] fetch 21 legal factor labels for case classification. Mackaay et al. [15] extract $\mathrm{N}$-grams features which creates by clustering semantically similar $\mathrm{N}$ grams. Sulea et al. [18] propose a SVM-based system, which uses the case description, time span and ruling as features. However, these methods only extract shallow text features or manual tags, which are difficult to collect on larger datasets. Therefore, when the amount of the data is large, they will not perform well.

Recently, owning to success of deep neural network in the natural language processing (NLP), computer vision (CV) and speech fields, some works begin to apply the deep neural network to the charge prediction tasks and show a huge performance boost. Luo et al. [14] propose an hierarchical attentional network method, which predicts charges and extracts relevant articles jointly. $\mathrm{Hu}$ et al. [3] propose an attention-based neural model by incorporating several discriminative legal attributes. The method proposed in this paper is classified into a deep neural network method.

Our work is also related to the task of text classification. Recently, various neural network (NN) architectures such as Convolutional Neural Networks (CNN) [6] and Recurrent Neural Networks (RNN) have been used for text classification. Zhao et al. [24] explore capsule network with dynamic routing for text classification. From the perspective of using the capsule network, our work is related to Zhao et al. [24].

Our model is loosely inspired from $\mathrm{Hu}$ et al. [3], they introduce several discriminative attributes of charges that provide additional information for few-shot charges. Compared with this line of works, although our work also handle the problem of fewshot charges, our work is different from their works, since (1) the strategy of our SECaps model in dealing with the problem of few-shot charges is different from the above (2) as far as we know, we are the first to introduce capsule network for charge prediction and achieve the state-of-the-art performance in charge prediction task.

\section{Conclusion}

In this paper, we focus on the few-shot problem of charge prediction according to the fact descriptions of criminal cases. To alleviate the problem, we propose a Sequence Enhanced Capsule model for charge prediction. In particular, our SECaps model employs the seq-caps layer, which can capture characteristics of the sequence and abstract advanced semantic features simultaneously, and then combine with focal loss, which can handle the unbalanced problem of charges. Experiments on the real-world datasets show that our SECaps model achieves $69.4 \%, 69.6 \%, 79.5 \%$ Macro F1 on three datasets respectively, surpassing existing state-of-the-art methods by a considerable margin. 


\section{References}

1. He, K., Zhang, X., Ren, S., Sun, J.: Deep residual learning for image recognition. In: Proceedings of the IEEE conference on computer vision and pattern recognition. pp. 770-778 (2016)

2. Hochreiter, S., Schmidhuber, J.: Long short-term memory. Neural computation 9(8), 17351780 (1997)

3. Hu, Z., Li, X., Tu, C., Liu, Z., Sun, M.: Few-shot charge prediction with discriminative legal attributes. In: Proceedings of the 27th International Conference on Computational Linguistics. pp. 487-498 (2018)

4. Katz, D.M., Nd, B.M., Blackman, J.: A general approach for predicting the behavior of the supreme court of the united states. Plos One 12(4) (2014)

5. Keown, R.: Mathematical models for legal prediction. Computer/lj 2, 829 (1980)

6. Kim, Y.: Convolutional neural networks for sentence classification. Eprint Arxiv (2014)

7. Kingma, D.P., Ba, J.: Adam: A method for stochastic optimization. arXiv preprint arXiv:1412.6980 (2014)

8. Kort, F.: Predicting supreme court decisions mathematically: A quantitative analysis of the right to counsel cases. American Political Science Review 51(1), 1-12 (1957)

9. Le, Y., Wang, Z.J., Quan, Z., He, J., Yao, B.: Acv-tree: A new method for sentence similarity modeling. In: IJCAI. pp. 4137-4143 (2018)

10. Lin, T.Y., Goyal, P., Girshick, R., He, K., Dollár, P.: Focal loss for dense object detection. IEEE transactions on pattern analysis and machine intelligence (2018)

11. Liu, C.L., Chang, C.T., Ho, J.H.: Case instance generation and refinement for case-based criminal summary judgments in chinese *. Journal of Informationence \& Engineering 20(4), 783-800 (2008)

12. Liu, C.L., Hsieh, C.D.: Exploring phrase-based classification of judicial documents for criminal charges in chinese. In: International Conference on Foundations of Intelligent Systems. pp. 681-690 (2006)

13. Liu, Y.H., Chen, Y.L., Ho, W.L.: Predicting associated statutes for legal problems. Information Processing \& Management 51(1), 194-211 (2015)

14. Luo, B., Feng, Y., Xu, J., Zhang, X., Zhao, D.: Learning to predict charges for criminal cases with legal basis pp. 2727-2736 (2017)

15. Mackaay, E., Robillard, P.: Predicting judicial decisions: The nearest neighbour rule and visual representation of case patterns. Computer/l.j pp. 302-331 (1974)

16. Mikolov, T., Sutskever, I., Chen, K., Corrado, G.S., Dean, J.: Distributed representations of words and phrases and their compositionality. In: Advances in neural information processing systems. pp. 3111-3119 (2013)

17. Nagel, S.S.: Applying correlation analysis to case prediction. Tex.l.rev 42(7), 1006-1017 (1964)

18. Octavia Maria Sulea, Marcos Zampieri, M.V., Genabith, J.V.: Exploring the use of text classication in the legal domain. In Proceedings of ASAIL workshop (2017)

19. Sabour, S., Frosst, N., Hinton, G.E.: Dynamic routing between capsules. In: Advances in neural information processing systems. pp. 3856-3866 (2017)

20. Salton, G., Buckley, C.: Term-weighting approaches in automatic text retrieval. Information processing \& management 24(5), 513-523 (1988)

21. Suykens, J.A., Vandewalle, J.: Least squares support vector machine classifiers. Neural processing letters 9(3), 293-300 (1999)

22. Wan-Chen Lin, Tsung-Ting Kuo, T.J.C.C.A.Y.C.J.C., de Lin, S.: Exploiting machine learning models for chinese legal documents labeling, case classification, and sentencing prediction. In Processdings of ROCLING (2014) 
23. Wang, D., Liu, Q.: An optimization view on dynamic routing between capsules. In: 6th International Conference on Learning Representations 2018 (2018)

24. Zhao, W., Ye, J., Yang, M., Lei, Z., Zhang, S., Zhao, Z.: Investigating capsule networks with dynamic routing for text classification (2018) 